\title{
Subtypes of Nonsocial Play: Comparisons Between Young Children With and Without Developmental Delays
}

\author{
Michael J. Guralnick, Mary A. Hammond, and Robert T. Connor \\ Center on Human Development and Disability, University of Washington
}

\begin{abstract}
Subtypes of nonsocial play were examined for matched groups of young typically developing children and children with mild developmental (cognitive) delays. Based on intercorrelation patterns, interindividual stability, and associations with peer interaction measures, findings indicated that the nonsocial play of children with delays can be characterized as multidimensional in a manner similar to that of typically developing children. However, analyses suggest different interpretations of the psychological meaning of nonsocial play subtypes for both groups of children than indicated by previous work. Context factors did not influence nonsocial play subtypes. Evidence was found indicating considerable heterogeneity within subtypes, suggesting the need for longitudinal studies focusing on children's peer-related social competence, which may reveal subgroups within subtypes that both refine the constructs and provide direction for clinical interventions.
\end{abstract}

Children who exhibit high levels of nonsocial play during unstructured activities with peers in early childhood settings are considered to be at risk for a variety of concurrent and later adjustment problems (Rubin, Bukowski, \& Parker, 1998; Rubin \& Mills, 1988). Yet, it has become evident that nonsocial play (i.e., playing alone when in the presence of available peers) is a complex multidimensional construct composed of distinct subtypes that appear to represent different behavioral patterns, developmental pathways, and developmental outcomes (Coplan, 2000; Coplan, Rubin, Fox, Calkins, \& Stewart, 1994; Harrist, Zaia, Bates, Dodge, \& Pettit, 1997; Rubin, 1982; Rubin, Stewart, \& Coplan, 1995). For otherwise typically developing children, at least three subtypes of nonsocial play have been identified consistently: reticent, solitary-passive, and solitary-active. Salient characteristics of each of these subtypes are described next.

The reticent behavior subtype consists of a behavioral pattern in which nonsocial play is char- acterized by frequent episodes of watching peers but not joining in their play and/or remaining unoccupied with no obvious purpose to the child's behavior. This pattern is correlated with anxious behaviors in social situations, and concurrent associations with temperamental shyness and internalizing problems have been reported (Coplan, 1998, 2000; Coplan et al., 1994; Coplan, Gavinski-Molina, Lagacé-Séguin, \& Wichmann, 2001; Coplan \& Rubin, 1998). Reticence is also associated with teacher ratings of lower social competence (Coplan, Gavinski-Molina et al., 2001). From a motivational perspective, Asendorpf (1990) has suggested that reticent behaviors reflect an approach-avoidance conflict. That is, children exhibiting these behaviors are interested in interacting with peers but at the same time are fearful of those interactions.

In contrast, children who engage in substantial levels of solitary-passive play apparently prefer to be alone, exploring and playing constructively with objects but are quite capable of socially com- 
petent interactions with peers when called upon to do so (Coplan et al., 1994; Coplan \& Rubin, 1998; Rubin, 1982). The solitary-passive subtype of nonsocial play is not associated with maladaptive behavior in early childhood, although there is some suggestion that problems may emerge over time (Rubin \& Mills, 1988). Recent work, however, suggests that solitary-passive behavior is associated with ratings of poorer academic ability and internalizing problems for boys but not for girls, with a similar trend for social competence (Coplan, Gavinski-Molina et al., 2001). Nevertheless, solitary-passive behavior is generally not considered to be a concern during early childhood, as children displaying this behavior pattern appear to be well-regulated emotionally (Rubin, Coplan, Fox, \& Calkins, 1995).

The third and final subtype that has been identified refers to a behavioral pattern in which children engage in functional activities (i.e., repetitious sensorimotor behaviors) or dramatic play when playing alone, although they have ready access to peers (Coplan et al., 1994). This solitaryactive form of play has been associated with maladaptive behaviors during early childhood, particularly impulsivity and externalizing problems (Coplan \& Rubin, 1998; Coplan et al., 1994; Rubin, 1982). Lower levels of teacher-rated social competence also have been reported for children exhibiting this behavioral pattern compared to children who do not display any solitary-active behavior (Coplan, Wichmann, \& Lagacé-Séguin, 2001). Although clearly interested in playing with others, children exhibiting this behavioral pattern may lead peers to reject or ignore them, thereby resulting in more frequent episodes of playing alone (see Rubin \& Asendorpf, 1993).

The subtyping of nonsocial play for typically developing children has considerably advanced our understanding of children's peer relationships, providing insights into motivational, social-cognitive, and other characteristics of each subtype. In addition, this more differentiated view may be of particular value in selecting children at greatest risk for peer interaction difficulties to participate in clinical interventions and in adjusting the content of those interventions in accordance with patterns corresponding to each subtype.

Of importance, and the focus of this study, despite increased levels of nonsocial play and peer competence difficulties of children with developmental (cognitive) delays in comparison to typically developing children, no attempt has been made to apply this multidimensional approach to this or any other group of children exhibiting developmental delays or disabilities. Generally, children with developmental delays exhibit higher levels of solitary play but lower levels of sustained play (group play) with peers (Guralnick, Connor, Hammond, Gottman, \& Kinnish, 1996; Guralnick \& Groom, 1987a, 1987b; Kopp, Baker, \& Brown, 1992). These play patterns presumably reflect various difficulties in peer-related social competence that have been identified (Guralnick, 1999a) and place children with delays at increased risk for later social isolation (Howell, Hauser-Cram, \& Warfield, 2001; Taylor, Asher, \& Williams, 1987; Williams \& Asher, 1992). If a similar multidimensional structure for nonsocial play applies to this group of children, information on subtypes may be especially informative for those conducting clinical interventions (Guralnick, 2001b).

Each of the three subtypes of nonsocial play is typically operationalized using Rubin's (2000) Play Observation Scale. Observations of children's play with peers is carried out during unstructured play, and children's interactions across a series of consecutive intervals are coded with respect to the major social participation categories (unoccupied, onlooking, solitary play, parallel play, conversation, and group play) as well as the quality of play when it occurs (functional, exploratory, dramatic, constructive, games with rules). Reticent behavior is defined as intervals consisting of unoccupied and/or onlooking behavior; solitary-passive behavior is defined as intervals consisting of solitary-exploratory and/or solitaryconstructive play; and solitary-active behavior is defined as intervals consisting of solitary-functional and/or solitary-dramatic play. This approach to defining nonsocial play subtypes was followed in this study to compare matched groups of typically developing children and children with mild developmental delays who participated in a series of 2-week playgroups with initially unfamiliar peers. In this study, we expected that similar levels of heterogeneity in nonsocial play and similar developmental processes were operating for both groups of children. Indeed, despite some unusual patterns, the peer relationships of children with developmental delays can be best understood within a developmental framework as similar developmental sequences, the nature of underlying processes, and factors influencing development appear to be relevant (Cicchetti \& Cohen, 1995; Guralnick, 1999a, 2001b; Hauser-Cram, Warfield, Shonkoff, \& Krauss, 2001). Accordingly, we ex- 
pected that distinct nonsocial play subtypes would be apparent for children with and without developmental delays.

In addition, assuming evidence for subtypes for both developmental status groups, we examined specific child characteristics and peer interaction patterns for each nonsocial play subtype in this study to determine their consistency with expectations of the "psychological meaning" associated with each subtype. Of special interest were measures related to emotional regulation and social competence with peers as suggested by research on typically developing children. Specifically, behavior problem measures of internalizing and externalizing were included given their expected associations with the reticent and solitaryactive subtypes, respectively. Moreover, to evaluate the quality and extent to which children whose behavioral patterns were associated with each of the three subtypes actually interacted with peers, we carried out a series of observations to assess the success of children's interactions with peers, their frequency of initiations, frequency of positive and negative behavior directed toward peers, the proportion of positive interactions, and the responsiveness of children to the positive social bids of their peers. Child characteristic measures of cognitive, language, and adaptive behavior also were included because these developmental status measures may be associated with the different subtypes, especially for children with developmental delays.

Based on the presumed psychological meanings of each subtype and previous correlational analyses of specific play categories associated with each subtype (Rubin, 1982), we expected that different patterns of peer-related social behavior based on these measures would result. Although many patterns are possible, children exhibiting more solitary-active behaviors would be expected to be less positive (proportion) and less successful in their social bids to peers, whereas those exhibiting more reticent and solitary-passive behaviors would be expected to initiate interactions with peers less frequently. We also expected that for children exhibiting more solitary-passive behaviors, no associations with indices of peer competence, such as the success of their social bids, would be apparent. Increased participation in any of the subtypes should be associated with less peer-directed positive or negative behavior. Again, consistent with an overall developmental framework, similar patterns were expected for both developmental status groups.

(C) American Association on Mental Retardation
The context of play, particularly the characteristics of play partners, can exert a considerable influence on children's play interactions, including nonsocial play (Coplan, 2000). Although many factors are relevant, such as chronological age-CA (Bailey, McWilliam, Ware, \& Burchinal, 1993) and friendship status of potential playmates (Hinde, Titmus, Easton, \& Tamplin, 1985), the developmental status of one's peers is also of consequence. From the perspective of children with developmental delays, the availability of typically developing children in play settings, especially mates of the same CA, generally increases children's levels of social interactions (Field, Roseman, DeStefano, \& Koewler, 1981; Guralnick et al., 1996). Whether the frequency of occurrence of the various subtypes of nonsocial play also can be altered by the availability of typically developing peers is an important issue that may have considerable clinical significance. In addition, it may be influential during continuing educational and policy discussions about the inclusion or mainstreaming of typically developing children and children with developmental, especially cognitive, delays in early childhood settings (Guralnick, 2001a). For typically developing children, the presence of children with delays may also have an impact on nonsocial play, as the overall peer interactions of children with developmental delays tends to increase as a consequence of their participation in inclusive settings (Guralnick et al., 1996). To examine this context issue, we arranged for children in each of the two developmental status groups to participate in settings containing children similar or different in developmental status. Specifically, playgroups consisted only of typically developing children or children with developmental delays as well as playgroups in which children from both developmental status groups participated. Each of the three nonsocial play subtypes was evaluated as a function of setting and developmental status group.

An additional context factor that can influence nonsocial play is the familiarity of the peers in the setting (Coplan, 2000). In fact, social interactions with peers increases substantially as children become acquainted with one another (Doyle, Connolly, \& Rivest, 1980; Shea, 1981). Nevertheless, the multidimensional structure of nonsocial play is evident in playgroups in which children are unfamiliar with one another (Coplan et al., 1994) and when children are together in the same preschool for substantial periods of time (Coplan, Gavinski-Molina et al., 2001; Coplan \& 
Rubin, 1998). Moreover, short-term stability for each of the three subtypes of nonsocial play has been found when correlating patterns at the beginning of a play session with those at the end of a play session for unfamiliar peers (Coplan et al., 1994). Additional preliminary work suggests stability over a 6-week period as well as some longer term stability of the three subtypes (Coplan, 2000). In the current study, playgroups of initially unfamiliar children were observed over a 2-week period, and the stability of the nonsocial play subtypes over that time period was evaluated. We anticipated that both developmental status groups would show similar levels of stability. Finally, changes over time for each nonsocial play subtype within each developmental status group were evaluated, and any shifts in the proportion of time engaged in each of the subtypes was expected to be similar across developmental status groups. We further anticipated that less reticent behavior would be apparent over time, as children adjusted to their new environment and playmates (Asendorpf, 1991).

In summary, the multidimensional nature of nonsocial play was investigated to determine its applicability to young children with mild developmental delays. Comparisons with typically developing children provided an opportunity to evaluate similarities and differences of nonsocial play subtypes. Our major hypothesis was that distinct nonsocial play subtypes would be apparent for children with and without developmental delays, with similar correlates to emotional regulation and peer social competence measures. Also investigated for both groups was whether peer interactions during unstructured play and related measures were compatible with the presumed psychological meaning of each subtype, whether the presence of play partners with diverse characteristics influenced the expression of the subtypes, and whether subtypes were stable over time, despite increasing familiarity with the environment and peers in the setting.

\section{Method}

\section{Overview}

Previously unacquainted groups of children were brought together to form a series of 12 separate playgroups ( $N=6$ children per playgroup). Children participating in the playgroups differed in terms of their developmental characteristicsreferred to as the developmental status variable (i.e., children with developmental delays or typi- cally developing children). The social environment of the playgroups also varied-referred to as the setting variable (i.e., playgroups consisting only of other children with similar developmental characteristics [homogeneous] or those in which children from both developmental status groups participated [inclusive]).

Of the 12 playgroups, 6 were homogeneous: 3 consisted of only typically developing children and 3 were composed of only children with developmental delays. The remaining 6 playgroups were inclusive; each consisted of 4 typically developing children and 2 children with developmental delays. As described later, a matching procedure ensured that typically developing children assigned to inclusive or homogeneous playgroups as well as children with developmental delays assigned to either playgroup were equivalent within each of the two types of playgroups in terms of child characteristic measures (CA, cognitive ability, language, adaptive behavior, and behavior problems). A similar matching process ensured equivalence across all groups for family demographic measures (social status, marital status). For each 2-week playgroup, the social and play interactions of each child were recorded during a designated free-play period.

\section{Participants}

Typically developing children were recruited through direct contact with administrators and teachers in public and private nursery schools and daycare programs. Children with developmental (cognitive) delays were recruited from community-based preschool programs and from rosters of children who received clinical evaluations from diagnostic clinics. The CA range for all participants was established at 4.25 to 5.50 years. Only boys were selected to participate in the playgroups because resources were not available to include gender as an additional independent variable, and more boys with delays were available in community preschools. Similarly, to avoid potential confounds due to race, only we selected Caucasian children. In addition, children were excluded from participating for any of the following reasons: (a) three siblings within 3 years of age of the child being considered, (b) teacher reports of major disruptive behavior problems, (c) legal blindness or major uncorrected hearing loss, (d) significant motor problems, (e) acquaintance with other children in the playgroup, and (f) living with the primary caregiver less than one year.

For selection and matching purposes, the re- 
vised version of the Wechsler Preschool and Primary Scale of Intelligence-WPPSI-R (Wechsler, 1989) was administered individually to all prospective participants. Full Scale IQs (FSIQs) as well as performance (PIQs) and verbal (VIQs) were obtained. Two language tests also were administered individually to each child. First, the revised version of the Test for Auditory Comprehension of Language (Carrow-Woolfolk, 1985) was administered. This test consists of scales for word classes and relations, grammatical morphemes, and elaborated sentences. A total score (standard score) is also obtained. Second, to supplement the receptive language assessment of the Test for Auditory Comprehension of LanguageRevised, we administered the expressive components of the Preschool Language Scale (Zimmerman, Steiner, \& Pond, 1979). Because of the lack of standardization, only raw scores were used (range $=0$ to 48 for verbal ability and 0 to 23 for articulation).

In addition to direct assessments of children's cognitive and language development, mothers served as respondents for assessments of their child's adaptive behavior and behavior problems. First, trained interviewers administered the Vineland Adaptive Behavior Scales Survey Form (Sparrow, Balla, \& Cicchetti, 1984) to mothers. Standard scores were obtained for each of the four domains (Communication, Daily Living Skills, Socialization, and Motor Skills) as well as for the total adaptive behavior score (mean alpha $=.88$, range $=.85$ to .90 ). Second, the mother's assessment of her child's behavior problems was based on the Child Behavior Checklist (Achenbach \& Edelbrock, 1991). Mothers rated the frequency of different behavior problems from a 118 -item questionnaire using a 3-point scale. Only the broad band internalizing and externalizing scales (alphas $=.65$ and .84 , respectively) $T$ scores in conjunction with a total behavior problem score were used for subject selection and matching purposes. Higher scores indicate greater perceived behavior problems. Finally, responses to a parent questionnaire provided basic demographic information. The Hollingshead Four Factor Index of Social Status (Hollingshead, 1975) was used to calculate a measure of family status (range $=8$ to 66).

Beyond the inclusionary and exclusionary criteria applied to all children as previously noted, specific criteria also were established for each of the two groups of children differing in developmental characteristics. Specifically, typically developing children were included if they achieved an FSIQ between 90 and 130. Children were excluded, however, for any of the following reasons: (a) VIQ or PIQ lower than 90, (b) Test for Auditory Comprehension of Language-Revised total score less than 90, (c) Child Behavior Checklist total problem score greater than the 90th percentile, (d) enrolled in a preschool program in which more than $15 \%$ of the children had established disabilities, or (e) had a sibling with an established disability. Similarly, children with developmental delays were included if they achieved an FSIQ between 52 and 80 . Children in this group were excluded, however, for any of the following reasons: (a) PIQ greater than 90, (b) Child Behavior Checklist total problem score greater than the 98th percentile or teacher reports of continuous and substantial disruption, and (c) a Test for $\mathrm{Au}-$ ditory Comprehension of Language-Revised total score less than 55 or greater than 90 .

\section{Matching Procedures}

Children with developmental delays were first identified for each playgroup, with typically developing children participating in both inclusive and homogeneous groups subsequently recruited from the same neighborhoods to maximize similar demographic characteristics. Children were tested on a continuous basis across a 4-year period, and playgroups were formed when an appropriate number of children meeting criteria were recruited. Homogeneous and inclusive playgroups were interspersed over the 4 years. On occasion, a child meeting established criteria was not included if his test scores were inconsistent with matching projections for the demographic and child characteristic measures.

As a result of this process, child characteristic measures were equivalent for the typically developing children participating in the inclusive and homogeneous playgroups. The only exception was that typically developing children participating in homogeneous playgroups had a higher Vineland Daily Living Skills score than did typically developing children participating in the inclusive playgroups, $p<.05$. Equivalent scores also were obtained across all child characteristic measures for children with developmental delays participating in homogeneous and inclusive settings.

As expected, significant differences were obtained for most of the child characteristic measures when comparing typically developing children and those with developmental delays (see Table 1 for details). The only exceptions were child's CA, the PIQ-VIQ discrepancy, and the Child Be- 
Table 1. Child Characteristic by Group

\begin{tabular}{|c|c|c|c|c|}
\hline \multirow[b]{2}{*}{ Characteristics $^{a}$} & \multicolumn{2}{|c|}{$\begin{array}{l}\text { Developmentally delayed } \\
\qquad(N=30)\end{array}$} & \multicolumn{2}{|c|}{$\begin{array}{l}\text { Typically developing } \\
\qquad(N=42)\end{array}$} \\
\hline & Mean & $S D$ & Mean & $S D$ \\
\hline CA (months) & 58.30 & 4.81 & 56.83 & 3.79 \\
\hline \multicolumn{5}{|l|}{ WPPSI-R } \\
\hline Full Scale IQ ${ }^{b}$ & 66.77 & 9.14 & 110.81 & 10.69 \\
\hline Performance IQ ${ }^{\mathrm{b}}$ & 68.83 & 10.10 & 110.00 & 11.56 \\
\hline Verbal IQ & 70.57 & 9.39 & 109.12 & 11.53 \\
\hline Performance-Verbal IQ & -1.73 & 10.18 & 0.88 & 14.03 \\
\hline Full Scale $M A^{b}$ & 38.45 & 6.44 & 62.58 & 7.56 \\
\hline Performance $\mathrm{MA}^{\mathrm{b}}$ & 39.70 & 7.14 & 62.10 & 7.71 \\
\hline Verbal MA ${ }^{b}$ & 40.58 & 6.35 & 61.64 & 7.99 \\
\hline \multicolumn{5}{|l|}{ TACL-R } \\
\hline Total Scores ${ }^{b}$ & 74.40 & 10.16 & 107.43 & 8.84 \\
\hline Word Class \& Relations ${ }^{b}$ & 77.93 & 15.85 & 108.79 & 11.02 \\
\hline Grammatical Morphemes ${ }^{\mathrm{b}}$ & 75.50 & 11.77 & 104.33 & 10.20 \\
\hline Elaborated Sentences ${ }^{b}$ & 82.03 & 9.37 & 107.79 & 12.15 \\
\hline \multicolumn{5}{|l|}{ PLS } \\
\hline Verbal Ability ${ }^{b}$ & 20.20 & 8.34 & 36.69 & 4.53 \\
\hline Articulation ${ }^{b}$ & 13.82 & 6.07 & 20.56 & 2.51 \\
\hline \multicolumn{5}{|l|}{ Vineland } \\
\hline Total Adaptive Behavior ${ }^{b}$ & 68.63 & 8.42 & 94.52 & 10.75 \\
\hline Communication ${ }^{b}$ & 76.67 & 11.93 & 96.29 & 8.68 \\
\hline Daily Living Skills & 71.67 & 9.53 & 92.33 & 11.80 \\
\hline Socialization ${ }^{b}$ & 79.73 & 10.57 & 98.95 & 9.54 \\
\hline Motor Skillsb & 67.13 & 13.97 & 97.40 & 13.04 \\
\hline \multicolumn{5}{|l|}{$\mathrm{CBCL}$} \\
\hline Total Behavior Problems ${ }^{b}$ & 53.43 & 8.16 & 47.14 & 6.86 \\
\hline Externalizing & 48.93 & 8.55 & 47.81 & 8.33 \\
\hline Internalizing & 47.93 & 8.66 & 45.31 & 7.39 \\
\hline
\end{tabular}

aWPPSI-R = Wechsler Preschool and Primary Scale of Intelligence; TACL-R = Test of Auditory Comprehension-Revised; PLS = Preschool Language Scale; Vineland $=$ Vineland Adaptive Behavior Scales; CBCL $=$ Child Behavior Checklist. bIndicates a significant difference using $t$ tests, $p<.05$, between typically developing children and children with developmental delays.

havior Checklist externalizing and internalizing factors. Finally, for family demographics, 91.7\% of the mothers were partnered, with an average family social strata based on the Hollingshead index of 2.15 (medium business, minor professional). The groups (developmental status or inclusive vs. homogeneous) did not differ on these two measures, which were analyzed with the chisquare and Kruskal-Wallace one-way analysis of variance, respectively.

\section{Playgroup Setting and Procedure}

Each 6-child playgroup operated 2.5 hours per day, 5 days per week, for 2 weeks (10 sessions) in either a morning or afternoon time period. Children arrived in separate vehicles via parents or drivers, and parents were asked to avoid contact with the other families or children for the duration of the playgroup. Parents were paid $\$ 100$ plus transportation expenses.

Playgroups were supervised by a teacher and
(C) American Association on Mental Retardation 
graduate assistant in a specially designed laboratory playroom. Children participated in a series of group and individual activities typical of preschool programs, including circle time, music, art, snack, and story. During two daily 30 -minute freeplay periods, children had access to the extensive array of toys and equipment found in the playroom. Separate areas provided opportunities for housekeeping, blocks, puzzles, games, and precast and manipulative toy play activities as well as an option for individual reading. Although the teacher generally encouraged social and play interactions among the children in other activities, during free-play periods the teacher limited her interactions to providing assistance when necessary.

Children's social and play interactions were videorecorded using split-screen technology: two remote controlled cameras mounted at either end of the playroom and a hand-operated camera in an adjacent observation room. The child being recorded at the time (focal child) wore a specially designed lightweight vest equipped with a professional quality wireless microphone and transmitter secured in a hidden pocket in the back of the vest. Other microphones were placed discreetly throughout the room and a control panel of mixers balanced the auditory signals.

Each child was observed for a total of $60 \mathrm{~min}-$ utes during free play over the 2 -week period. Recording commenced on the second playgroup day and was divided into segments of 10 consecutive minutes for each of 6 recording periods per child. The order of recording children was randomized within blocks of six 10-minute segments, and no child was observed more than once per day. In addition, recordings were distributed such that each child was videotaped on three occasions within the first week (Time 1) and on three occasions during the second week (Time 2).

\section{Observational Measures \\ Social participation and cognitive play}

A time code superimposed on each videotape in conjunction with a remotely controlled tapestop device allowed observers to view tapes at 10second intervals. Coders recorded the categories of social participation and level of cognitive play where required during each 10 -second interval using the Peer Observation Scale developed by Rubin (1985). Nonsocial play subtypes were derived from information obtained from the this scale, which consists of 10 mutually exclusive and exhaustive categories. The first 3 were derived from
Parten's (1932) social participation categories, consisting of the following play classifications: (a) solitary (playing alone), (b) parallel (playing next to another child), and (c) group (playing with another child; a combination of Parten's associative and cooperative play categories). Nested within these three social participation categories are four measures of cognitive play based on the work of Smilansky (1968): (a) functional (simple repetitive play), (b) constructive (learns to use materials, creates something), (c) dramatic (role-taking and pretend play), and (d) games with rules (child behaves in accordance with prearranged rules). If any 10second interval was coded as either solitary, parallel, or group play, then one of the four cognitive play categories was also scored.

The 7 remaining categories consisted of the following: (a) unoccupied behavior (child not playing), (b) onlooker behavior (child watches other children but does not enter into play), (c) reading or listening (reading, leafing through a book, listening to a tape), (d) exploration (examining physical properties of objects as a solitary activity), (e) $a c$ tive conversation (talking, questioning, and suggesting to other children but not playing), (f) transition (moving from one activity to another), and (g) adult-directed (any activity with an adult).

Peer Observation Scale based measures

Based on the Peer Observation Scale, the proportion of intervals for each of the three nonsocial play subtypes for each child was calculated. This was carried out separately for each of the two observation periods (Time 1, Time 2), generating 180 coded intervals per child for each time point. Reticent behavior was indexed by summing the proportion of intervals children engaged in unoccupied and/or onlooking behaviors. Solitary-passive behavior was indexed by summing the proportion of intervals children engaged in solitary-exploratory and/or solitary-constructive play. Finally, solitary-active behavior was indexed by summing the proportion of intervals children engaged in solitary-functional and/or solitary-dramatic play.

\section{Individual social behaviors}

Each videotape was reviewed a second time in order to examine specific peer-related social behaviors. For this purpose, the Individual Social Behavior Scale was developed based on the work of White and Watts (1973) and has been adapted for use with children who have delays (e.g., Guralnick \& Groom, 1987b). Specifically, observers record continuously the occurrence of individual social behaviors for all defined categories. The fol- 
lowing categories were designed to record social interactions of the focal child as directed to peers: (a) seeks attention of peer; (b) uses peer as a resource; (c) leads in peer activities-direct, positive, or neutral; (d) leads in peer activities-indirect, positive, or neutral; (e) leads in peer activitiesdirect, negative; (f) leads in peer activities-indirect, negative; (g) imitates a peer; $(\mathrm{h})$ joins peers in specific activity; (i) verbally supports peer's statement; (j) verbally competes with peer; $(k)$ shows pride in product to peer; (l) expresses affection to peer; (m) shows empathy toward peer; (n) expresses hostility toward peer; (o) takes unoffered object; and (p) seeks agreement from peer.

With the exception of the involved observation and defends property categories, each of the focal child individual social behaviors just listed was classified as to whether it was an initiation. A focal child initiated event is one in which no prior verbal or nonverbal interaction occurred for at least 3 seconds.

Ten of the remaining categories focused on the social behaviors of the focal child in response to directed activities of peers. Categories consisted of following the lead of a peer ( 2 categories tied to direct/indirect and positive/neutral dimensions), failing to follow the lead of a peer ( 2 categories as above), responding and failing to respond to a peer's attempt to use the focal child as a resource (2 categories), responding and failing to respond to a peer's attention-seeking behavior ( 2 categories), and responding and failing to respond when a peer sought agreement from the focal child (2 categories).

Seven of the categories designed to record the social interactions of the focal child as directed to peers ( $a$ through $f, p$ ) also were judged as either successful or unsuccessful. Definitions for successful or unsuccessful social interactions were specific to each social behavior category. For example, the gains the attention of peer category would be coded as successful if the peer attended within 5 seconds, either visually or verbally, or moved closer to or touched the focal child. The response of the peer must be appropriate to the attention-getting effort of the focal child.

Coders were free to review any segment of the tape as often as needed. The coding protocol was divided into 30-second intervals following the time codes superimposed on the tape. Although coding was continuous, these divisions provided a structure for the coding task and served as a framework for establishing reliability.
Peer interaction composites. Measures of interest were the success of social bids (proportion), total initiations, positive behavior total directed toward peers (joins, verbal support, verbal competition, pride in product, expresses affection, shows empathy, lead peer direct and indirect positive or neutral, use of peer as resource, imitation, seek attention of peer, and seek agreement from peer), negative behavior total directed to peers (expresses hostility, lead peer direct and indirect negative, and takes unoffered object), the proportion of positive interactions directed to peers, and the extent to which the target child was responsive to the positive social bids of peers. To obtain the responsiveness measure, we calculated the number of instances in which the target child responded positively (positively responding to the lead peer direct and indirect, use as a resource, seeking attention, and seeking agreement). We also determined the number of times the target child failed to respond positively to the social bids in those categories. Responsiveness was defined as the proportion of positive responses to the total.

\section{Reliability}

Prior to coding, five raters were trained for a period of 12 to 19 weeks on the two observation scales. Videotapes of pilot playgroups were used for training and final prestudy reliability assessments. Following the training program, all raters achieved the minimum average criterion necessary for participation of $70 \%$ interobserver agreement for each of the major categories for ten 10-minute segments from a reliability tape containing complex segments for each of the two scales. Reliability also was obtained for each rater during the course of the study for $25 \%$ of the playgroup tapes selected on a random basis but balanced to ensure representation from the two types of social settings, groups, and time.

For the Social Participation and Cognitive Play Scale, reliability was based on percentage agreement obtained across each of the 10 -second observation intervals (number of agreements divided by the total number of observations and transformed to a percentage). Cohen's (1960) kappa also was calculated where appropriate. For prestudy reliability, raters agreed on a mean of $84 \%$ (range $=83 \%$ to $85 \%)$ of the intervals, $\kappa=.80$, for the 10 categories of the Social Participation Scale. Using only those instances in which observers agreed that a cognitive play coding was required, we found an average interobserver agreement of $94 \%$ (range $=93 \%$ to $96 \%$ ) for the 4 
cognitive play categories. During the course of the study, average interobserver agreement continued to be high in all instances for each of the 12 groups: social participation, $86 \%$ (range $=82 \%$ to $90 \%), \kappa=.81$ (range $=.76$ to .85 ); cognitive play, $91 \%$ (range $=82 \%$ to $97 \%$ ).

For the Individual Social Behavior Scale, raters were considered to be in agreement if codes matched within a specified 10 -second interval using the "best fit" matching method (Hollenbeck, 1978). Rules for aligning coding protocols are designed to maximize best fits within an event-based system. (A reliability manual describing this method is available from the first author.) In addition to the individual social behavior categories, a nointeraction event was included to complete the possible options within each interval. Percentage agreement was obtained for each 10-minute segment by taking the total number of agreements, dividing by the total number of observed individual social interactions, and transforming to a percentage. Calculated in this manner, the average prestudy agreement for this scale was $85 \%$ (range $=84 \%$ to $87 \%), \kappa=.75$. Given agreement on the occurrence of a particular social interaction, observers further agreed on an average of $82 \%$ (range $=80 \%$ to $90 \%$ ) of the occasions as to whether the event could be classified as successful or unsuccessful and an average of 79\% (range = $67 \%$ to $88 \%$ ) as to whether or not selected focal child behaviors were initiations. Mean reliabilities for observations carried out during the course of the study (25\% of the total) were as follows: individual social behaviors, $87 \%$ (range $=83 \%$ to $92 \%), \kappa=.78$ (range $=.76$ to .83 ); successful/ unsuccessful, $91 \%$ (range $=84 \%$ to $100 \%$ ); and initiations, $80 \%$ (range $=67 \%$ to $96 \%$ ).

\section{Results}

\section{Effects of Setting}

Setting effects were examined first for each of the three nonsocial play subtypes for each of the two developmental status groups separately. Mixed design ANOVAs were performed with two levels of setting (homogeneous vs. inclusive) and repeated measures for the time factor (Time 1 vs. Time 2). These analyses yielded nonsignificant results for setting and for setting by time interactions. Consequently, for subsequent analyses, homogeneous and inclusive groups within each developmental status group were combined.

(C) American Association on Mental Retardation

\section{Distribution Among Subtypes}

Descriptive statistics for the three nonsocial play subtypes for the two developmental status groups for each time period are presented in Table 2. Overall, the proportion of intervals children engaged in the three subtypes of nonsocial play was .52. As expected, children with delays engaged in more nonsocial play $(M=.58)$ than did children without delays $(M=.45), t(70)=4.24, p<.001$. The relatively high overall level of nonsocial play may be related to the unfamiliarity of the peers and the setting and, perhaps, the difficulties posed by having 6 children in the playgroup. Solitarypassive play occurred most frequently for both developmental status groups, with reticent behavior and solitary-active behavior occurring equally often.

Mixed design ANOVAs were carried out for each nonsocial play subtype with two levels of developmental status group and repeated measures for the two levels of time. Reticent behavior did not vary between groups or across time nor was the interaction significant. Despite the fact that children in both groups engaged in the same amount of reticent behavior, more unoccupied behavior was evident in the group of children with delays $(M=.07)$ than without delays $(M=$ $.04), t(70)=2.21, p<.05$. For the solitary-passive subtype, significant effects for group, $F(1,70)=$ 11.28, $p<.01$, and time, $F(1,70)=4.93, p<$ .05 , were obtained, but the interaction was not significant. As seen in Table 2, the group of children with delays engaged in more solitary-passive play than did the typically developing group, and solitary-passive behavior decreased over time for both groups. In contrast, solitary-active behavior increased over time, $F(1,70)=8.08, p<.01$, but no other effects were significant. Although the overall amount of solitary-active behavior did not differ between the two groups, children with delays exhibited more solitary-functional play, $t(70)$ $=2.26, p<.05$. However, on an absolute basis, this latter difference was quite small.

\section{Intercorrelations Among Nonsocial Play Subtypes}

A series of Pearson product-moment correlations was calculated separately for each developmental status group at both Times 1 and 2. As seen in Table 3, none of the subtypes for either of the groups was significantly positively correlated with one another at either time. Although two 
differences between developmental status groups in the intercorrelation matrices approached significance, $p<.10$, for (a) reticent, Time 1 and solitary-active, Time 1 and (b) solitary-active, Time 1 and solitary-passive, Time 2, none of the tests for differences between independent correlations were significant between groups. Accordingly, this replicates Coplan et al.'s (1994) findings suggesting distinct nonsocial play subtypes for typically developing children and extends their results to children with developmental delays.

\section{Stability}

The stability of each subtype for individual children within each developmental status group is also displayed in Table 3. As can be seen, significant correlations between Times 1 and 2 were found for all three subtypes for both developmental status groups. Reticent behavior was especially stable for both groups of children.

\section{Relationships With Child Characteristics and Social Behavior With Peers}

A series of multiple regressions were carried out to examine hypothesized associations between nonsocial play subtypes and both child characteristics and social behavior with peers. This multiple regression approach enabled us to examine the unique relationships between the nonsocial play subtypes and both child characteristics and the peer interaction measures while controlling for developmental status groups. In addition, interaction terms were used to determine the degree of similarity of the patterns of association between developmental status groups.

First, child characteristics were examined (see Table 1) based on WPPSI-R (Full Scale IQ and Full Scale MA), the Preschool Language Scale verbal ability measure, Test of Auditory Comprehension-Revised total, Vineland total, and the internalizing and externalizing scores of the Child Be- havior Checklist. A separate analysis of the family social status measure was also carried out. Eight hierarchical multiple regressions were performed in which each of the seven child characteristic measures (and the one family status measure) was regressed on (a) a dummy-coded delay/typical child variable, (b) the three subtypes of nonsocial play behaviors (total proportions), and (c) three interactions between each subtype and the delayed/typical variable. Results revealed no significant associations (betas) for Steps 2 and 3 for any variable.

Next, a similar analysis was carried out for a series of measures based on the Individual Social Behavior Scale combining the two time periods: successful bids (proportion), total initiations, positive behavior to peers (total), negative behavior to peers (total), positive to peers (proportion), and responsive to peers (proportion). Specifically, six hierarchical multiple regressions were performed in which each of the peer-related social behavior measures was regressed on (a) a dummy-coded delayed/typical variable, (b) the three subtypes of nonsocial play behaviors (total proportions), and (c) three interactions between each subtype and the delayed/typical variable. We note that at Step 3 the addition of the interactions between each subtype of nonsocial play behaviors and developmental status group was not statistically significant for any of the dependent variables. These results indicate similar patterns of associations between subtypes of nonsocial play behaviors and peer-related social behaviors for both the developmental status groups. Because the interactions were not significant, the multiple regression results at Step 2 are shown in Table 4.

At Step 1, developmental status group showed significant positive associations with total positive behavior to peers, beta $=.55, p<.001$, the proportion of positive behavior to peers, beta

Table 2. Descriptive Statistics (Proportions) for Nonsocial Play Subtypes by Group and Time

\begin{tabular}{|c|c|c|c|c|c|c|c|c|c|c|c|c|}
\hline \multirow[b]{3}{*}{ Subtype } & \multicolumn{6}{|c|}{$\begin{array}{l}\text { Developmentally delayed } \\
\qquad(N=30)\end{array}$} & \multicolumn{6}{|c|}{$\begin{array}{l}\text { Typically developing } \\
\qquad(N=42)\end{array}$} \\
\hline & \multicolumn{2}{|c|}{ Time 1} & \multicolumn{2}{|c|}{ Time 2} & \multicolumn{2}{|c|}{ Total } & \multicolumn{2}{|c|}{ Time 1} & \multicolumn{2}{|c|}{ Time 2} & \multicolumn{2}{|c|}{ Total } \\
\hline & Mean & $S D$ & Mean & $S D$ & Mean & $S D$ & Mean & $S D$ & Mean & $S D$ & Mean & $S D$ \\
\hline Reticent & .14 & .10 & .14 & .11 & .14 & .10 & .11 & .07 & .12 & .08 & .12 & .07 \\
\hline Solitary-passive & .32 & .17 & .26 & .11 & .29 & .13 & .21 & .14 & .19 & .10 & .20 & .09 \\
\hline Solitary-active & .13 & .13 & .18 & .13 & .15 & .11 & .12 & .09 & .14 & .09 & .13 & .08 \\
\hline
\end{tabular}


$=.39, p<.01$, and the proportion of responsiveness to peers, beta $=.28, p<.05$. In all instances, typically developing children had higher mean scores on the peer interaction measures than did children with delays.

At Step 2, the entry of the three subtypes of nonsocial play behaviors showed significant changes for all of the peer-related social behaviors except for total initiations and the proportion of responsiveness to peers. For the subtypes, as shown in Table 4, reticent behavior was significantly negatively related to three of the peer-related social measures: the proportion of successful bids, total positive behavior to peers, and total negative behavior to peers. Solitary-passive behavior was significantly negatively related to four of the peer-related social measures: total initiations, total positive behavior to peers, total negative behavior to peers, and proportion of positive behavior to peers. Finally, solitary-active behavior was significantly negatively related to two of the peer-related social measures: total positive behavior to peers and total negative behavior to peers. Also at Step 2, developmental status group continued to show significant positive associations with total positive behavior to peers and the proportion of positive behavior to peers. Changes in $R^{2}$ for the six dependent variables at Step 2 were as follows: successful bids (proportion), .11, $p<$ .05 ; initiations (total), .10, $p>.05$; positive be- havior to peers (total), .27, $p<001$; negative behavior to peers (total), .20, $p<.01$; positive to peers (proportion), .09, $p<.05$; and responsive to peers (proportion), .05, $p>.05$.

\section{Discussion}

Our findings reveal that the nonsocial play of young children with mild developmental (cognitive) delays can be characterized as multidimensional in a manner similar to that of typically developing children. Analyses of the intercorrelation matrices provide evidence for distinct subtypes of nonsocial play-reticent, solitary-passive, and solitary-active. For both groups of children, these subtypes were either uncorrelated or negatively associated with one another and showed considerable stability over the 2-week period. Direct comparisons between groups also did not reveal any differences with respect to these correlational patterns. This similarity between developmental status groups was supported by further multiple regression analyses, which revealed a common pattern of associations between nonsocial play subtype behaviors and peer interaction measures. Accordingly, our results replicate previous work with typically developing young children (Coplan et al., 1994) and extend them to young children with mild developmental delays.

At the same time, important differences from

Table 3. Intercorrelations Among Nonsocial Play Subtypes by Group and Time (1 or 2)

\begin{tabular}{lccccc}
\hline Group/Time & $\begin{array}{c}\text { Solitary- } \\
\text { passive-1 }\end{array}$ & $\begin{array}{c}\text { Solitary- } \\
\text { active-1 }\end{array}$ & Reticent-2 & $\begin{array}{c}\text { Solitary- } \\
\text { passive-2 }\end{array}$ & $\begin{array}{c}\text { Solitary- } \\
\text { active-2 }\end{array}$ \\
\hline $\begin{array}{l}\text { Developmentally delayed } \\
\text { Reticent-1 }\end{array}$ & & & & & \\
Solitary-passive-1 & -.16 & $-.40^{*}$ & $.83^{* * *}$ & -.31 & $-.43^{*}$ \\
Solitary-active-1 & & -.04 & -.06 & $.58^{* * *}$ & .05 \\
Reticent-2 & & -.36 & .17 & $.39^{*}$ \\
Solitary-passive-2 & & & & -.28 & $-.52^{* *}$ \\
Solitary-active-2 & & & & & -.01 \\
Typically developing & & & & & \\
Reticent-1 & -.22 & .01 & $.69 * * *$ & .09 & -.16 \\
Solitary-passive-1 & & -.12 & $-.34 *$ & $.34 *$ & .20 \\
Solitary-active-1 & & & -.16 & -.27 & $.52^{* * *}$ \\
Reticent-2 & & & -.05 & $-.34^{*}$ \\
Solitary-passive-2 & & & & & -.02 \\
Solitary-active-2 & & & & & \\
\hline
\end{tabular}

$* p<.05 .{ }^{* *} p<.01 . * * * p<.001$.

(C) American Association on Mental Retardation 
previous work emerged with respect to the possible meaning of the subtypes of solitary-passive and reticent behavior and their clinical implications. For solitary-passive behavior, as noted earlier, available evidence for typically developing children suggests that this form of nonsocial play is generally benign during the preschool years, primarily indicating a preference for solitary play but with children quite capable of socially competent interactions as dictated by social circumstances (e.g., Rubin, 1982). The negative association with total positive behaviors appears to reflect the fact that these children were simply less active socially overall (as was the case for the other subtypes). However, in addition to the negative association with socially interactive behavior toward peers (both positively and negatively), solitary-passive behavior was negatively associated with the frequency of initiations and the proportion of positive social behaviors. This suggests that solitarypassive behavior may be related to peer interaction difficulties, perhaps associated with specific social skills deficits. Accordingly, the solitary-passive subtype of nonsocial play may well be associated with peer competence difficulties, thereby requiring clinical intervention during the preschool period. Interestingly, a recent report by Coplan, Gavinski-Molina et al. (2001) suggests that low social competence as rated by teachers is associated with solitary-passive play for typically developing children, but only for boys. As noted earlier, only boys were involved in the present study. More detailed assessments of children engaging in varying levels of solitary-passive play focusing on measures of peer-related social competence, especially patterns occurring during social tasks relating to peer group entry, conflict res- olution, and maintaining play, will be required to confirm this hypothesis regarding the connection between peer social skills deficits and solitary-passive behavior (see Guralnick, 1999a). Similarly, longitudinal studies are needed to determine whether solitary-passive behavior is associated with later social isolation and feelings of loneliness as has been reported for older children with developmental delays (e.g., Howell et al., 2001). Perhaps subgroups can be identified in this manner who are at greater risk of later problems.

Our findings also suggest that the construct of reticent behavior may need to be evaluated more closely. As expected, the more reticent behavior children engaged in, the less socially interactive behavior (both positive and negative) they displayed. In addition, reticent behavior was significantly negatively correlated with the proportion of success of social bids. This pattern of findings also suggests possible social skill difficulties. That is, reticent behavior may not only be a consequence of a motivational style (i.e., approachavoidance conflict) but related as well to social skills deficits. Early studies of typically developing children indicate that reticent behavior does not appear to be associated with social competence (Asendorpf, 1990), but more recent findings do suggest this as a possibility (Coplan, Gavinski-Molina et al., 2001). In our study we were unable to examine the meaning of this construct as primarily tied to motivational or emotional regulation issues because observational measures closely tied to the reticence construct, such as hovering (Coplan et al., 1994), were not obtained. More comprehensive observational and related measures are clearly needed and may reveal even greater heterogeneity for children with and without delays.

Table 4. Summary of Results of the Hierarchical Multiple Regressions for the Peer Interaction Measures

\begin{tabular}{lcccc}
\hline & & \multicolumn{3}{c}{ Subtypes of nonsocial play behaviors } \\
\cline { 3 - 5 } Peer interaction measures & Group & Reticent & Solitary-passive & Solitary-active \\
\hline Successful bids (proportion) & .16 & $-.25^{*}$ & .20 & -.20 \\
Initiations (total) & -.07 & -.18 & $-.32^{*}$ & -.16 \\
Positive behavior to peers (total) & $.28^{* *}$ & $-.29^{* *}$ & $-.50^{* * *}$ & $-.32^{* *}$ \\
Negative behavior to peers (total) & -.06 & $-.37^{* *}$ & $-.30^{*}$ & $-.37^{* *}$ \\
Positive to peers (proportion) & $.32^{* *}$ & .16 & $-.26^{*}$ & .08 \\
Responsive to peers (proportion) & .19 & -.01 & -.16 & -.17 \\
\hline
\end{tabular}

Notes: Results are shown for Step 2 of the hierarchical multiple regression analyses. Values are standardized regression coefficients (betas).

${ }^{a}$ Delayed versus typical.

$* p<.05 . * p<.01 . * * p<.001$. 
Indeed, longitudinal studies suggest considerable heterogeneity for the reticent subtype even for typically developing children (see Asendorpf, 1994; Coplan, 2000).

Contrary to expectations, child characteristic and behavior problem measures were not correlated with the reticent or solitary-passive subtypes for either group. This is not too surprising for child characteristics related to cognition, language, and even adaptive behavior because previous associations have been extremely weak (Rubin, 1982). The absence of associations with mother-rated internalizing behavior for the reticent subtype is, however, inconsistent with findings from previous research (e.g., see Coplan, 2000; Coplan \& Rubin, 1998). Perhaps the fact that our study involved maternal ratings of behavior problems rather than teacher ratings, as in previous work, is responsible for this inconsistency. Assuming that teacher ratings are based primarily on child behavior in the play setting, stronger associations are to be expected than when ratings are based on observations in diverse settings as is the case for maternal ratings. Moreover, other recent work also failed to confirm the association between internalizing behavior and the reticent subtype (Coplan, Gavinski-Milina, et al., 2001), suggesting the possibility that reticence may be a marker for difficulties in social competence. As noted above, the association between reticent behavior and the peer social interaction measure of successful bids is consistent with this hypothesis.

Similarly, the solitary-active subtype did not show the expected association with externalizing behavior for either developmental status group. Perhaps the fact that we excluded children with significant behavior problems in this study contributed to this result. Even so, however, this association with externalizing behavior has not been particularly robust in previous research with typically developing children (Coplan \& Rubin, 1998), especially boys (Coplan, Gavinski-Molina et al., 2001). Unfortunately, our peer interaction measures did not contribute to further elucidating the meaning of this subtype. Children who participated in more solitary-active play engaged in less positive and negative social behavior with peers, but this was a general and expected pattern, which did not differ from the other nonsocial play subtypes. More comprehensive and detailed assessments of peer interactions, particularly in the context of the social task of conflict resolution, may help refine the behavioral characteristics of this group further.

In addition, consistent with Coplan, Wichmann et al. (2001), no cognitive or language differences were associated with the solitary-active subtype. Again, identical patterns were obtained for children with and without delays. Of note, solitary-active behavior occurred at a much higher frequency in this study compared to previous work. Because only boys were included in our sample, it is possible that this form of play is more gender-stereotyped, as suggested by Coplan, Gavinski-Molina et al., 2001. Indeed, solitary-active play increased over time. Differing interpretations of functional and dramatic play by coders in different studies may have contributed to this difference as well.

In this study, we also investigated the possible effects of context factors relating to familiarity and the developmental status of peers in the playgroups on the subtypes of nonsocial play. Comparisons between Times 1 and 2 revealed considerable interindividual stability. As the setting and peers became more familiar, small changes were detected as solitary-active behavior occurred more frequently over time, but solitary-passive play decreased. In this relatively brief time period, no overall changes in nonsocial play were found. It would be expected, however, that with longer time periods, familiarity and experience would exert an effect and perhaps allow patterns of interaction to become further differentiated within subtypes (see Asendorpf, 1994; Coplan, 2000).

For the context factor relating to the influence of the developmental status of one's peers, no effects on nonsocial play subtypes were obtained for either group. It may well be that the factors governing the patterns of nonsocial play may be more closely linked to characteristics such as child temperament or dimensions of social competence. This hypothesis is consistent with previous results suggesting that although the frequency of social behaviors can be altered by the developmental status of one's playmates (e.g., Guralnick et al., 1996), measures more representative of peer-related social competence, such as peer group entry and conflict resolution, are not affected (see Guralnick, 1999b). This highlights once again the importance of developing comprehensive intervention programs carefully tailored to individual child characteristics, prevailing patterns of social interaction, and processes presumed to be govern- 
ing peer-related social competence (Guralnick, 2001b).

In summary, previous research focusing on typically developing children has established the multidimensional nature of nonsocial play and advanced our understanding of important developmental constructs as well as provided direction for clinical interventions. As revealed in this initial study of the characteristics of nonsocial play of young children with developmental delays, the potential for similar conceptual advances and clinical utility clearly exists for this population as well. Indeed, evidence was found in this study suggesting that children with developmental delays display a multidimensional structure in their nonsocial play that is similar to that of typically de-

Nevertheless, for this potential to be realized, a better understanding of the psychological meaning of nonsocial play subtypes will be required. The identification of subgroups within subtypes may prove to be most informative in future work, especially when strong consideration is given to measures of peer-related social competence. Although perhaps more pressing for children with delays, identifying subgroups within subtypes may further our understanding of the psychological meaning of the subtypes for typically developing children as well. As suggested by recent work (e.g., Coplan, Gavinski-Molina et al., 2001), additional correlates, especially those related to social competence, are helping to refine the meaning of these subtypes and will perhaps lead to the identification of other subtypes as haps best illustrated by the finding in the present study that $20 \%$ of the children with developmental delays and $23 \%$ of the typically developing children were at or above the 67th percentile on two or more of the measures defining each nonsocial play subtype. This degree of heterogeneity must be taken into account in future studies. Moreover, the significant negative associations between reticent and solitary-active behaviors obtained both in the current study and that of Coplan et al. (1994) also suggest that a re-evaluation of the distinct nature of these constructs is warranted. Correspondingly, focused efforts to measure peer-related social competence in the context of longitudinal studies examining the development of children's peer relationships should enable investigators to establish the relative risks for peer interaction difficulties for chilveloping children. well (Harrist et al., 1997). This complexity is per-

dren with and without delays displaying differing nonsocial play patterns and provide guidance for the content of clinical interventions. The fact that unoccupied behavior contributed more to the reticent behavior subtype for children with delays offers the possibility that longitudinal research may even reveal differences between developmental status groups over time. Taken together, this greater degree of differentiation will likely bring us closer to a more complete understanding of the complexities of nonsocial play for all children.

\section{References}

Achenbach, T. M., \& Edelbrock, C. (1991). Manual for the Child Behavior Checklist/4-18 and 1991 Profile. Burlington: University of Vermont, Department of Psychiatry.

Asendorpf, J. B. (1990). Development of inhibition during childhood: Evidence for situational specificity and a two-factor model. Developmental Psychology, 26, 721-730.

Asendorpf, J. B. (1991). Development of inhibited children's coping with unfamiliarity. Child Development, 62, 1460-1474.

Asendorpf, J. B. (1994). The malleability of behavioral inhibition: A study of individual developmental functions. Developmental Psychology, 30, 912-919.

Bailey, D. B., Jr., McWilliam, R. A., Ware, W. B., \& Burchinal, M. A. (1993). Social interactions of toddlers and preschoolers in same-age and mixed-age play groups. Journal of Applied Developmental Psychology, 14, 261-276.

Carrow-Woolfolk, E. (1985). Manual for the Test for Auditory Comprehension of Language-Revised. Allen, TX: DLM Teaching Resources.

Cicchetti, D., \& Cohen, D. J. (1995). Perspectives on developmental psychopathology. In D. Cicchetti \& D. J. Cohen (Eds.), Developmental psychopathology: Vol. 1. Theories and methods (pp. 3-20). New York: Wiley.

Cohen, J. (1960). A coefficient of agreement for nominal scales. Educational and Psychological Measurement, 20, 37-46.

Coplan, R. J. (1998). Social anxiety and maladjustment in the preschool. On-line Proceedings of the 5th Internet World Congress on Biomedical Sciences '98, McMaster University, Hamilton, Ontario, Canada. (Available from URL: http://www.mcmaster.ca/inabis98/ simantov/dus0133/index.html)
(C) American Association on Mental Retardation 
Coplan, R. J. (2000). Assessing nonsocial play in early childhood: Conceptual and methodological approaches. In K. Gitlin-Weiner, A. Sandgrund, \& C. Schaefer (Eds.), Play diagnosis and assessment (2nd ed., pp. 563-598). New York: Wiley.

Coplan, R. J., Gavinski-Molina, M.-H., Lagacé-Séguin, D. G., \& Wichmann, C. (2001). When girls versus boys play alone: Nonsocial play and adjustment in kindergarten. Developmental Psychology, 37, 464-474.

Coplan, R. J., \& Rubin, K. H. (1998). Exploring and assessing nonsocial play in the preschool: The development and validation of the preschool play behavior scale. Social Development, 7, 72-91.

Coplan, R. J., Rubin, K. H., Fox, N. A., Calkins, S. D., \& Stewart, S. L. (1994). Being alone, playing alone, and acting alone: Distinguishing among reticence and passive and active solitude in young children. Child Development, 65, 129-137.

Coplan, R. J., Wichmann, C., \& Lagacé-Séguin, D. G. (2001). Solitary-active play behavior: A marker variable for maladjustment in the preschool? Journal of Research in Childhood Education, 15, 164-172.

Doyle, A., Connolly, J., \& Rivest, L. (1980). The effect of playmate familiarity on the social interactions of young children. Child Development, 51, 217-223.

Field, T., Roseman, S., DeStefano, L., \& Koewler, J. H., III. (1981). Play behaviors of handicapped preschool children in the presence and absence of nonhandicapped peers. Journal of Applied Developmental Psychology, 2, 49-58.

Guralnick, M. J. (1999a). Family and child influences on the peer-related social competence of young children with developmental delays. Mental Retardation and Developmental Disabilities Research Reviewes, 5, 21-29.

Guralnick, M. J. (1999b). The nature and meaning of social integration for young children with mild developmental delays in inclusive settings. Journal of Early Intervention, 22, 7086.

Guralnick, M. J. (2001a). A framework for change in early childhood inclusion. In M. J. Guralnick (Ed.), Early childhood inclusion: Focus on change (pp. 3-35). Baltimore: Brookes.

Guralnick, M. J. (2001b). Social competence with peers and early childhood inclusion: Need for alternative approaches. In M. J. Guralnick (Ed.), Early childhood inclusion: Focus on change (pp. 481-502). Baltimore: Brookes.

Guralnick, M. J., Connor, R., Hammond, M., Gottman, J. M., \& Kinnish, K. (1996). Immediate effects of mainstreamed settings on the social interactions and social integration of preschool children. American Journal on Mental Retardation, 100, 359-377.

Guralnick, M. J., \& Groom, J. M. (1987a). Dyadic peer interactions of mildly delayed and nonhandicapped preschool children. American Journal of Mental Deficiency, 92, 178-193.

Guralnick, M. J., \& Groom, J. M. (1987b). The peer relations of mildly delayed and nonhandicapped preschool children in mainstreamed playgroups. Child Development, 58, 1556-1572.

Harrist, A. W., Zaia, A. F., Bates, J. E., Dodge, K. A., \& Pettit, G. S. (1997). Subtypes of social withdrawal in early childhood: Sociometric status and social-cognitive differences across four years. Child Development, 68, 278-294.

Hauser-Cram, P., Warfield, M. E., Shonkoff, J. P., \& Krauss, M. W. (2001). Children with disabilities. Monographs of the Society for Research in Child Development, 66(3, Serial No. 266).

Hinde, R. A., Titmus, G., Easton, D., \& Tamplin, A. (1985). Incidence of "friendship" and behavior toward strong associates versus nonassociates in preschoolers. Child Development, 56, 234-245.

Hollenbeck, A. R. (1978). Problems of reliability in observational research. In G. P. Sackett (Ed.), Observing behavior: Vol. 2. Data collection and analysis methods (pp. 79-98). Baltimore: University Park Press.

Hollingshead, A. B. (1975). Four factor index of social status. Unpublished manuscript. Yale University, Department of Sociology, New Haven.

Howell, A., Hauser-Cram, P., \& Warfield, M. E. (2001). Preschool predictors of later peer acceptance and loneliness in children with disabilities. Paper presented at the biennial meeting of the Society for Research in Child Development, Minneapolis.

Kopp, C. B., Baker, B. I., \& Brown, K. W. (1992). Social skills and their correlates: Preschoolers with developmental delays. American Journal on Mental Retardation, 96, 357-366.

Parten, M. B. (1932). Social participation among 
preschool children. Journal of Abnormal Social Psychology, 27, 243-269.

Rubin, K. H. (1982). Nonsocial play in preschoolers: Necessarily evil? Child Development, 53, 651-657.

Rubin, K. H. (1985). The Play Observation Scale (POS) (rev.) Waterloo, Ontario: University of Waterloo.

Rubin, K. H. (2000). The Play Observation Scale. University of Maryland, College Park: Author.

Rubin, K. H., \& Asendorpf, J. B. (1993). Social withdrawal, inhibition, and shyness in childhood: Conceptual and definitional issues. In K. H. Rubin \& J. B. Asendorpf (Eds.), Social withdrawal, inbibition, and shyness in childhood (pp. 3-17). Hillsdale, NJ: Erlbaum.

Rubin, K. H., Bukowski, W., \& Parker, J. G. (1998). Peer interactions, relationships, and groups. In W. Damon (Ed.) \& N. Eisenberg (Vol. Ed.), Handbook of child psychology: Vol. 3. Social, emotional, and personality development (5th ed., pp. 619-700). New York: Wiley.

Rubin, K. H., Coplan, R. J., Fox, N. A., \& Calkins, S. D. (1995). Emotionality, emotional regulation, and preschoolers' social adaptation. Development and Psychopathology, 7, 4962.

Rubin, K. H., \& Mills, R. S. L. (1988). The many faces of social isolation in childhood. Journal of Consulting and Clinical Psychology, 56, 916924.

Rubin, K. H., Stewart, S., \& Coplan, R. J. (1995). Social withdrawal in childhood: Conceptual and empirical perspectives. In T. H. Ollendick \& R. J. Prinz (Eds.), Advances in clinical child psychology (Vol. 17, pp. 157-196). New York: Plenum Press.
Shea, J. D. C. (1981). Changes in interpersonal distances and categories of play behavior in the early weeks of preschool. Developmental Psychology, 17, 417-425.

Smilansky, S. (1968). The effects of sociodramatic play on disadvantaged preschool children. New York: Wiley.

Sparrow, S. S., Balla, D. A., \& Cicchetti, D. V. (1984). Vineland Adaptive Behavior Scales. Circle Pines, MN: American Guidance Service.

Taylor, A. R., Asher, S. R., \& Williams, G. A. (1987). The social adaptation of mainstreamed mildly retarded children. Child Development, 58, 1321-1334.

Wechsler, D. (1989). Wechsler Preschool and Primary Scale of Intelligence-Rev. San Antonio: Psychological Corp.

White, B. L., \& Watts, J. C. (1973). Experience and environment (Vol. 1). Englewood Cliffs, NJ: Prentice-Hall.

Williams, G. A., \& Asher, S. R. (1992). Assessment of loneliness at school among children with mild mental retardation. American Journal on Mental Retardation, 96, 373-385.

Zimmerman, I. L., Steiner, V. G., \& Pond, R. E. (1979). Preschool Language Scale (Rev. ed.). Columbus, $\mathrm{OH}$ : Merrill.

Received 6/17/02, accepted 4/16/03.

Editor-in-charge: Frank Floyd

This research was supported by Grant H024K9002 from the Department of Education, Office of Special Education Programs. Requests for reprints should be sent to Michael J. Guralnick, Center on Human Development and Disability, Box 357920, University of Washington, Seattle, WA 98195-7920. E-mail: mjgural@u. washington.edu. 